\title{
Classificação de características produtivas fenotípicas de diferentes raças de poedeiras através da mineração de dados
}

\author{
Phenotypic production characteristics from different breeds of laying hens through data mining
}

\author{
Priscila Becker Ferreira $^{\mathrm{I}^{*}}$ Marcos Martinez do Vale ${ }^{\mathrm{II}}$ Andre Macedo ${ }^{\mathrm{III}}$ Lenise Shroder Boemo $^{\mathrm{I}}$ \\ Paulo Roberto Nogara Rorato ${ }^{\text {II }}$ Tamiris Barbosa Beck ${ }^{\mathrm{IV}}$
}

\section{RESUMO}

O estudo foi realizado com o banco de dados do Programa de Melhoramento Genético de Poedeiras de Ovos Marrons do Laboratório de Avicultura (LAVIC) do Departamento de Zootecnia da Universidade Federal de Santa Maria (UFSM), onde são utilizadas aves das raças Plymouth Rock White $(P R W)$, Plymouth Rock Barred $(P R B) e$ Rhode Island Red (RIR). Os dados utilizados foram referentes a duas gerações das três raças durante o primeiro ciclo de produção, dos anos de 2009 e 2010. Os parâmetros avaliados foram: taxa de postura semanal, peso dos ovos e peso corporal. $O$ estudo foi dividido em duas abordagens de análise, a primeira análise foi realizada com os dados produtivos com o objetivo de identificar pontos críticos de seleção fenotípica, relacionados à caracterização zootécnica, e a segunda abordagem consistiu na associação dos mesmos parâmetros produtivos, com os dados climáticos de Santa Maria - RS. Os dados foram analisados pela técnica de Mineração de Dados, sendo utilizada a tarefa de classificação com a construção de árvore de decisão pelo algoritmo J48. A mineração dos dados indicou a taxa de postura média na $25^{\underline{a}}$ semana de idade e posteriormente o peso médio dos ovos na $33^{a}$ semana de idade como as principais variáveis relacionadas com a diferenciação das raças estudadas, (precisão de 0,833). Na segunda abordagem, as árvores de classificação obtidas apresentaram precisão entre 0,88 e 0,91, sugerindo uma forte associação entre as variáveis ambientais e o desempenho produtivo das aves. Poedeiras PRW são mais sensíveis ao calor que as RIR e PRB e apresentam, na fase de pré-pico, uma maior taxa de postura. A Mineração de Dados permite classificar a produtividade das raças de poedeiras de ovos marrons, podendo indicar as influências ambientais sobre o fenótipo das diferentes raças estudadas.

Palavras-chave: bioclimatologia, ovos marrons, melhoramento animal, ambiência.

\begin{abstract}
The study was conducted with the database obtained from the Breeding Program of Brown Egg Laying Hens of the Poultry Laboratory (LAVIC) of the Department of Animal Science of the Federal University of Santa Maria (UFSM), where it is used poultry breeds such as White Plymouth Rock (PRW), Barred Plymouth Rock (PRB) e Red Rhode Island (RIR). The data used referred to two generations of these three breeds during the first production cycle of 2009 and 2010. The parameters evaluated were: weekly rate of egg laying, egg weight and body weight. The research was divided into two approaches of analysis, the first being performed with the production data, aiming to identify critical points of phenotypic selections related to zootechnical characterization, and the second approach consisted in the association of the same production parameters together with the weather data of Santa Maria - RS. The data was analyzed using the Data Mining technique, using the classification task by building the decision tree with the $\mathrm{J} 48$ algorithm. The data mining indicated the average rate of egg laying at 25 weeks of age and the average weight of eggs at 33 weeks of age, and pointed these as the main variables related to the differentiation of breeds studied, (accuracy of 83.3\%). On the second approach, the decision trees obtained showed accuracy between $88 \%$ and $91 \%$, suggesting a strong association between the environmental variables and the production performance of the birds. The PRW breed is more sensitive to heat than the RIR and the PRB breed and they showed a higher rate of production during the prepeak phase. The data mining allowed to classify the productivity of the brown egg laying hens, which may indicate environmental influences on the phenotype of the different breeds studied.
\end{abstract}

Key words: bioclimatology, brown eggs, animal breeding, environment.

\footnotetext{
IPrograma de Pós-graduação em Zootecnia, Departamento de Zootecnia, Universidade Federal de Santa Maria (UFSM), Campus Universitário, 97105-900, Santa Maria, RS, Brasil. E-mail: pri_zoot@hotmail.com. *Autor para correspondência.

IIDepartamento de Zootecnia, UFSM, Santa Maria, RS, Brasil.

II'Curso de Medicina Veterinária, UFSM, Santa Maria, RS, Brasil.

${ }^{\text {Iv }}$ Curso de Zootecnia, Universidade Federal do Pampa (UNIPAMPA), Dom Pedrito, RS, Brasil.
} 


\section{INTRODUÇÃO}

A principal orientação dos programas de melhoramento genético de poedeiras é aplicar um pacote de medidas que direcione as características de maior influência sobre a relação receita/custo para a melhoria constante no lucro da atividade comercial de produção de ovos. O valor genético-econômico de um plantel de aves reprodutoras é em função da taxa de postura, do tamanho do ovo, peso corporal, e ainda de outras características correlacionadas. Com isso, é importante a busca por novas metodologias de análise que possam contribuir para melhorar o processo de seleção de poedeiras para alcançar ganhos na produção de ovos por intermédio da identificação de características fenotípicas associadas ao ambiente (PIASSI et al. 1995).

O melhoramento tradicional, baseado na teoria da genética quantitativa, tem assegurado ganho genético contínuo de todas as características de produção em aves. A maior parte do progresso genético obtido nas características quantitativas tem sido decorrente da seleção baseada no fenótipo do animal, ou na estimativa do valor genético aditivo derivado do fenótipo(DEKKERS, 1999).

Dentre as técnicas de análise de bancos de dados, a Mineração de Dados é uma técnica promissora em várias áreas de conhecimento, envolvendo tarefas de classificação, associação ou agrupamento (FAYYAD \& STOLORZ, 1997; LAVRAC, 1999). A classificaçãoé uma tarefa com interessantes possibilidades de aplicação, devido à capacidade de extrair padrões representáveis graficamente por uma árvore de decisão ou classificação (BERRY \& LINOFF, 1997). Em avicultura, essa abordagem de análise de dados foi aplicada em estudos de ambiência e de processos produtivos com resultados promissores (VALE et al., 2008; VALE et al., 2010; LIMA et al., 2010), por outro lado, poucos pesquisadores aplicam esta técnica em melhoramento genético.

Na avicultura moderna, o ambiente climáticoé um dos principais causadores de perdas na produção animal, sendo que os extremos climáticos podem gerar severas perdas na produção, devido ao estresse por calor, e as mudanças climáticas podem impactar severamente, exigindo novas abordagens na seleção genética dos animais (NARDONE et al., 2006; NÄÄS et al., 2010). O estresse térmico é prejudicial às aves poedeiras, não somente pela mortalidade, mas também pela perda de condição corporal e quantidade de ovos que seriam produzidos, sendo importante que os geneticistas possam prever o impacto de fatores ambientes sobre a adaptação aoclima (PEREIRA et al., 2010).
O objetivo deste trabalho foi a caracterização zootécnica e identificação do impacto de fatores ambientais climáticos no desempenho produtivo de diferentes raças de galinhas poedeiras para a identificação de pontos críticos de seleção genética, por intermédio da técnica de Mineração de Dados.

\section{MATERIAL E MÉTODOS}

O estudo foi realizado com o banco de dados do Programa de Melhoramento Genético de Poedeiras de Ovos Marrons do Laboratório de Avicultura (LAVIC) do Departamento de Zootecnia da Universidade Federal de Santa Maria (UFSM), onde são produzidas aves das raças Plymouth Rock White (PRW), Plymouth Rock Barred (PRB) e Rhode Island Red (RIR). Os dados utilizados foram referentes a duas gerações das três raças durante o primeiro ciclo de produção, dos anos de 2009, nos meses de março a agosto, e em 2010, nos meses de fevereiro a julho.

As análises foram divididas em duas abordagens, a primeira referente ao desempenho zootécnico, realizada com os dados produtivos das três raças estudadas, com o objetivo de identificar pontos críticos de seleção fenotípica, relacionados à caracterização zootécnica das raças, em que se avaliaram os seguintes parâmetros (atributos): taxa de postura semanal (\%) da 22 a $32^{\mathrm{a}}$ semana de idade, peso corporal médio das aves da 27a a $32^{a}$ semana de idade e peso médio dos ovos da $23^{\mathrm{a}}$ a $43^{\mathrm{a}}$ semana de idade. Os valores médios semanais dos parâmetros das três raças estão apresentados na tabela 1 .

A segunda abordagem de análise consistiu na associação dos parâmetros zootécnicos com os dados climáticos de Santa Maria-RS, Brasil, provenientes do INMET (Instituto Nacional de Meteorologia, Brasil), a fim de identificar características da expressão fenotípica das raças ligadas ao clima. Foram organizados dois bancos de dados, sendo um para taxa de postura e outro para peso de ovos, classificados com relação à produtividade média esperada (baseada em dados de anos anteriores) como sendo produtividade NORMAL. As demais classes de produtividade INFERIOR e SUPERIOR foram determinadas pelos limites da classe NORMAL, estabelecida segundo a média mais ou menos um desvio padrão.

Os dados climáticos foram sumarizados a partir de um banco de dados meteorológico, correspondente à localidade e período de ocorrência dos parâmetros produtivos analisados. As variáveis climáticas diárias foram transformadas em médias, máximas e mínimas semanais para a temperatura, umidade relativa do ar e pressão atmosférica. Ao longo de cada 
Tabela 1 - Parâmetros produtivos das aves das diferentes raças, de março a setembro de 2009 e nos meses de fevereiro a julho do ano de 2010.

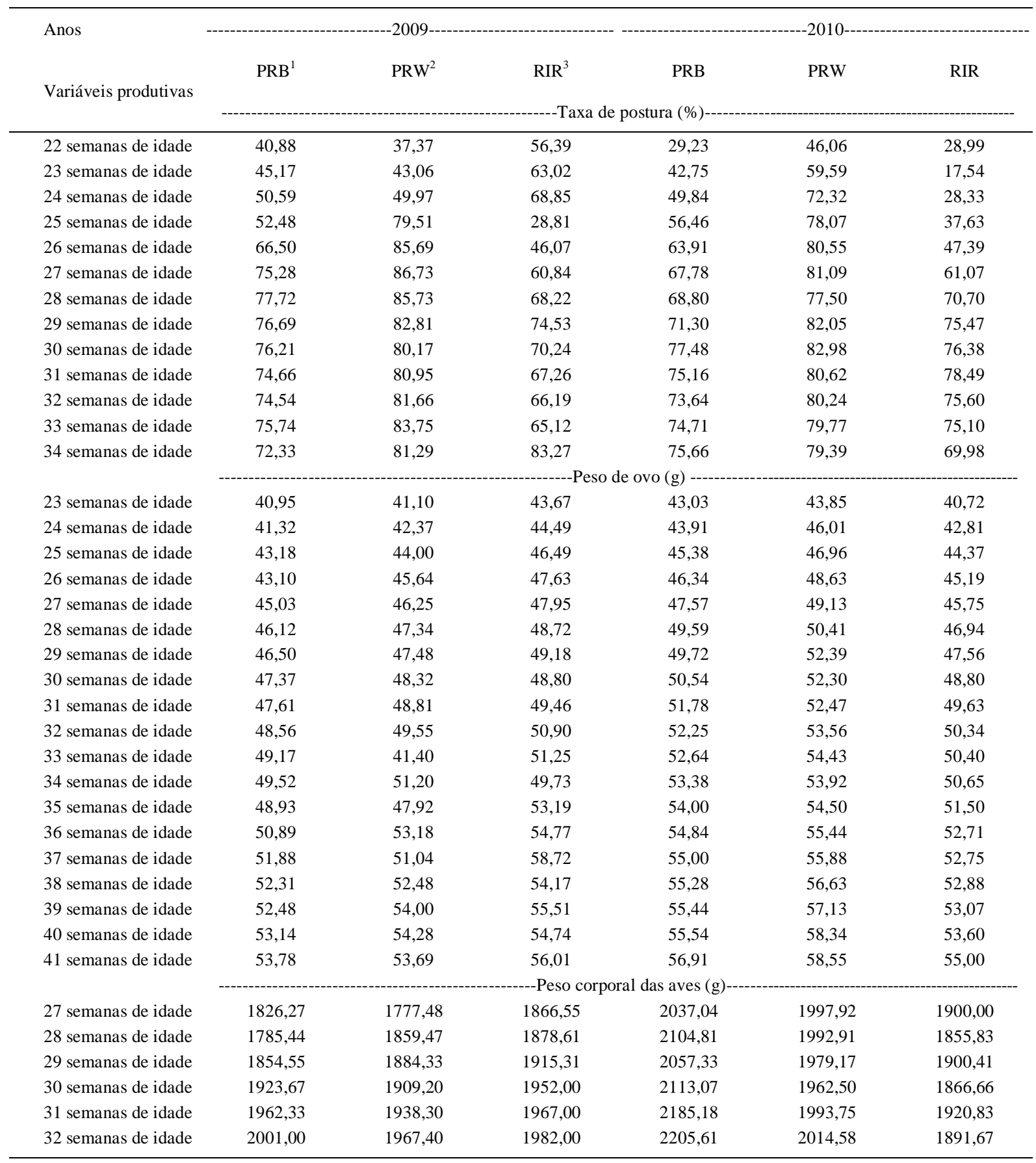

${ }^{1}$ Plymouth Rock Barred $;{ }^{2}$ Plymouth Rock White $\mathrm{e}^{3}$ Rhode Island Red.

semana produtiva, foi derivado o cálculo do número de dias na semana com temperaturas acima de 28,30 e $32^{\circ} \mathrm{C}$, ou inferiores a 10 e $12^{\circ} \mathrm{C}$, com o objetivo de identificar o impacto de extremos climáticos. As médias anuais das variáveis climáticas estão apresentadas na tabela 2 . Os dados sumarizados foram posteriormente integrados em sincronia com as datas das médias semanais dos bancos de dados de produção. 
Os dados foram analisados utilizando o programa computacional Weka ${ }^{\circledR}$ versão 3-4, aplicandose uma tarefa de classificação, com a construção de árvore de decisão. O algoritmo utilizado foi o J48, uma implementação do C4.5 para tarefas de classificação (QUINLAN, 1993; QUINLAN, 1996), que gera uma árvore de classificação, uma visualização gráfica na forma de uma árvore invertida, em que o nó raiz é a primeira variável com maior poder de classificação, apresentando abaixo de si os ramos, formados pelos demais atributos com as regras semânticas que permitem a classificação (folha). Para melhorar a precisão dos modelos, foi utilizado o balanceamento das classes, técnica de amostragem com reposição, aplicado pelo filtro Resample, do próprio programa Weka ${ }^{\circledR}$ (WITTEN \& FRANK, 2005), e a aplicação de técnicas de seleção de atributos.

Os modelos foram avaliados segundo as precisões conforme LAVRAC (1999), medidas em função das classificações corretas ou incorretas para o modelo e para a acurácia das classificações para cada uma das classes das raças PRW, PRB e RIR, na primeira abordagem, e para as categorias de produção NORMAL, INFERIOR e SUPERIOR para a relação com o clima, na segunda abordagem. As precisões e acurácias foram representadas entre 0 e 1 , sendo que 1 representa $100 \%$ de acerto do modelo. No estudo do impacto climático, os modelos foram avaliados e comparados entre si por três especialistas em avicultura, considerando como pré-requisito dos especialistas o mínimo de dois anos de experiência com avicultura e produção de ovos. Os especialistas avaliaram as regras geradas e elegeram as mais relevantes para o melhoramento genético e identificação de expressão de fenótipos.

\section{RESULTADOS E DISCUSSÃO}

Árvores de classificação são representações gráficas que permitem a extração de regras de classificação. A partir do nó raiz, podemos obter condicionais das variáveis em que são definidos os domínios de classificação, sendo representado na forma de uma regra semântica em pares condicionais tipo: SE <condicional> ENTÃO <classe> (VALE et al., 2010). Para figura 1-A, a primeira regra da esquerda pode ser descrita como: Regra $1=$ SE taxa de postura ao completar as 25 semanas $\leq 66,7 \%$ E taxa de postura ao completar as 25 semanas $\leq 44,12 \%$ E peso do ovo $\leq 45 \mathrm{~g}$ ENTÃO classe PRB, podendo ser resumida como: Regra $1=$ SE taxa de postura ao completar as 25 semanas $\leq 44,12 \%$ E peso do ovo $\leq 45 \mathrm{~g}$ ENTÃO classe PRB.

$\mathrm{Na}$ análise de desempenho zootécnico, a classificação das raças de poedeiras em função da produtividade, na mineração dos dados, gerou árvore de classificação para as raças conforme figura 1-A. A precisão do modelo foi de 0,833 , classificando as raças com 0,800 de acurácia para a classificação nas aves das raças PRW e PRB, e acurácia de 0,910 para a raça RIR. A taxa de postura média na $25^{\mathrm{a}}$ semana de idade foi o principal atributo capaz de classificar as raças em função do desempenho zootécnico. Atributos posicionados na raiz da árvore, conforme observado para a produção de ovos na 25 a semana de idade, apresentam um alto poder de classificação (WITTEN \& FRANK, 2005), sendo uma

Tabela 2 - Médias dos parâmetros climáticos de março a setembro de 2009 e de fevereiro julho do ano de 2010.

\begin{tabular}{|c|c|c|c|c|c|c|}
\hline \multirow[t]{2}{*}{ Variáveis climáticas } & \multicolumn{3}{|c|}{ 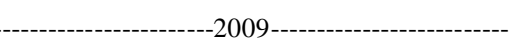 } & \multicolumn{3}{|c|}{$---2010---$} \\
\hline & MAX $^{1}$ & $\mathrm{MED}^{2}$ & $\mathrm{MIN}^{3}$ & MAX & MED & MIN \\
\hline Temperatura $\left({ }^{\circ} \mathrm{C}\right)$ & 15,83 & 15,24 & 14,67 & 18,38 & 17,83 & 17,32 \\
\hline Umidade relativa do ar (\%) & 83,37 & 80,92 & 78,38 & 84,08 & 81,81 & 79,34 \\
\hline Pressão atmosférica (hPA) & 1005 & 1005 & 1004 & 1005 & 1005 & 1004 \\
\hline Dias com temperatura $>25^{\circ} \mathrm{C}$ & 7,00 & 2,55 & 0,00 & 7,00 & 2,95 & 0,00 \\
\hline Dias com temperatura $>30^{\circ} \mathrm{C}$ & 3,00 & 0,64 & 0,00 & 5,00 & 1,05 & 0,00 \\
\hline Dias com temperatura $>32^{\circ} \mathrm{C}$ & 2,00 & 0,23 & 0,00 & 3,00 & 0,50 & 0,00 \\
\hline Dias com temperatura $<10^{\circ} \mathrm{C}$ & 7,00 & 3,23 & 0,00 & 6,00 & 1,55 & 0,00 \\
\hline Dias com temperatura $<5^{\circ} \mathrm{C}$ & 7,00 & 1,36 & 0,00 & 2,00 & 0,36 & 0,00 \\
\hline Dias com temperatura $<0^{\circ} \mathrm{C}$ & 2,00 & 0,14 & 0,00 & 0,00 & 0,00 & 0,00 \\
\hline
\end{tabular}

${ }^{1}$ média das máximas semanais; ${ }^{2}$ média das médias semanais $\mathrm{e}^{3}$ média das mínimas semanais. 


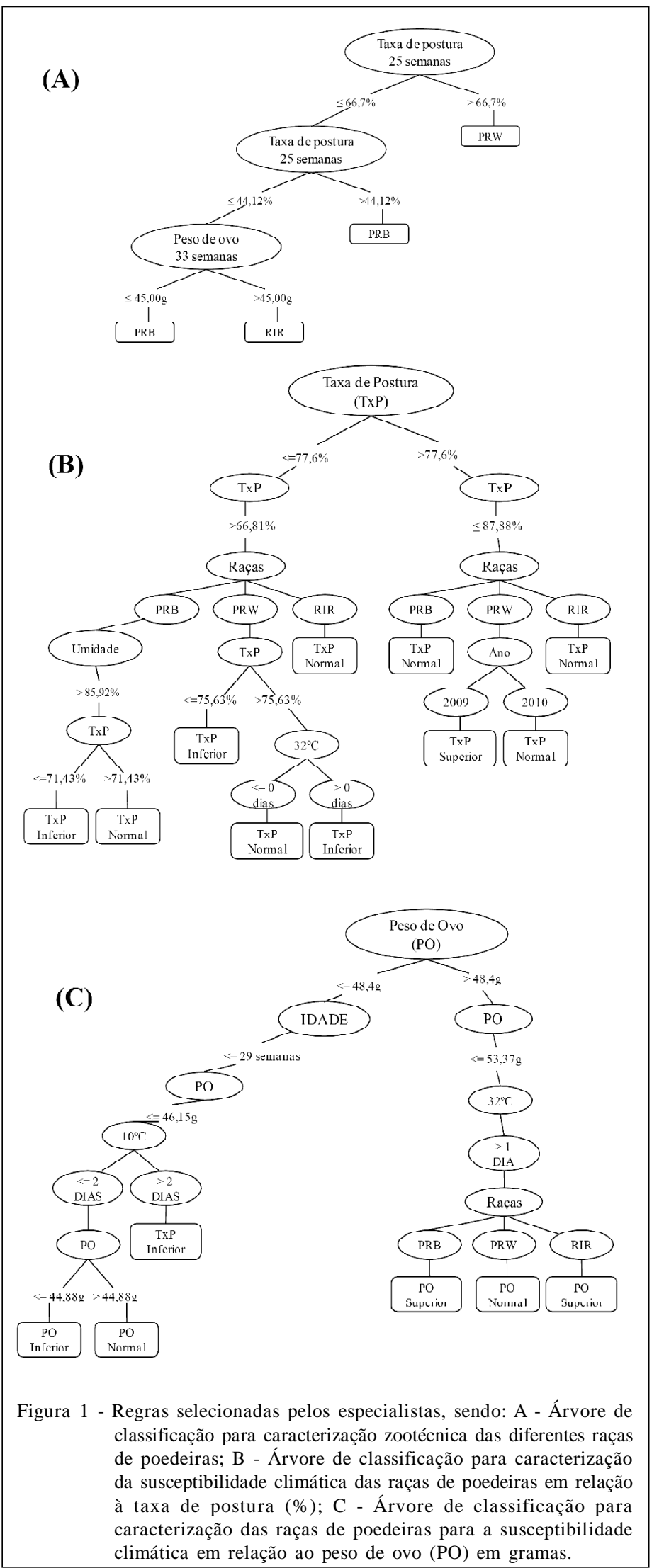

Ciência Rural, v.43, n.1, jan, 2013. 
variável importante para seleção fenotípica, quando o objetivo é manter a característica produtiva das raças ou identificar animais superiores. A seleção de aves de postura com base no desempenho parcial tem o intuito de aumentar a produção total de ovos, visando também a aumentar os ganhos genéticos por geração (POGGENPOELet. al., 1996).

A partir do modelo da figura 1-A, as aves PRW apresentaram, como característica para classificação, a taxa de postura superior a $66,7 \%$ na $25^{\text {a }}$ semana de idade. A raça PRB foi classificada zootecnicamente quando a taxa de postura ficou acima de $44,12 \%$ até $66,7 \%$ ou quando foi inferior a $44,12 \%$ e o peso médio de ovo foi inferior ou igual a $45 \mathrm{~g}$ na $33^{\mathrm{a}}$ semana de idade. As aves RIR foram caracterizadas pela taxa de postura inferior a $44,12 \%$ no período prépico e peso médio de ovo na 33르 semana superior a $45 \mathrm{~g}$. As variáveis obtidas pelo modelo da figura 1-A para classificação são de grande importância na seleção de aves, devido à alta herdabilidade, sendo de 0,29 para produção de ovos e de 0,75 para peso do ovo, para linhagens de poedeiras de ovos marrons (HAGGER, 1994).

A aplicação do modelo da figura 1-A pode gerar importantes ganhos nos processos de seleção fenotípica, aplicada no melhoramento de aves, pela automação da avaliação e redução do volume de dados a ser estudado, diminuindo custos computacionais e permitindo a sumarização de um conhecimento por intermédio da extração de regras (MITRA et al., 2002). Também pela possibilidade de identificação das aves com características superiores às médias das raças já na 33르 semana de idade, reduzindo os custos onerosos com testes de progênie até o final do ciclo de produção.

$\mathrm{Na}$ segunda abordagem de análise sobre o impacto climático, as árvores de classificação obtidas apresentaram precisão entre 0,880 e 0,912 , indicando uma forte relação entre as variáveis ambientais e o desempenho produtivo das aves. O pré-processamento dos dados, com a sumarização dos parâmetros climáticos semanais, permitiu identificar padrões climáticos que interferiram diferentemente na expressão produtiva das raças de poedeiras (Figura 1-B e C). As regras geradas nas duas abordagens realizadas foram selecionadas pelos especialistas conforme sua relevância e apresentados na tabela 3 .

As aves da raça PRW tiveram sua taxa de postura dentro da média esperada em 2010 e superior em 2009. Em contrapartida, as aves das raças RIR e PRB não diferenciaram sua taxa de postura conforme o ano, provavelmente devido à diferenciação na expressão fenotípica em relação ao clima. Odesempenho foi inferior
Tabela 3 - Precisão dos modelos (Prec) e acurácia (Acc) das regras das três abordagens de modelos de classificação de produtividade conforme o clima.

\begin{tabular}{lcc}
\hline & TxP $^{1}$ & $\mathrm{PO}^{1}$ \\
\hline Prec $^{2}$ & 0,880 & 0,912 \\
Inferior $^{2}$ & 0,758 & 0,786 \\
Normal $^{2}$ & 0,913 & 0,919 \\
Superior $^{2}$ & 0,853 & 1,000 \\
Total & 19 & 13 \\
Relevantes $^{3}$ & 10 & 6 \\
\hline
\end{tabular}

${ }^{1}$ Abordagens de análise. TxP: taxa de postura e PO: peso do ovo.

${ }^{2}$ Precisão (Prec) dos modelos e acurácia das regras (Acc) para as classes Inferior, Normal e Superior.

3 Julgamento de especialistas para a relevância das regras no melhoramento genético de poedeiras.

ao esperado pelas aves da raça PRW quando submetidas ao calor em dias com temperatura igual ou superior a $32^{\circ} \mathrm{C}$. Esse resultado pode justificar o pior desempenho da produção de ovos desta raça no ano de 2010, em relação ao ano de 2009, pois, em 2010, os somatórios das temperaturas médias e máximas e de dias com temperaturas acima de $28^{\circ} \mathrm{C}, 30^{\circ} \mathrm{Ce} 32^{\circ} \mathrm{C}$ foram superiores aos do ano de 2009 (Figura 1-B). As aves da raça PRW foram classificadas com produção de ovos superior. A alta produção de ovos vem sendo apontada como um dos principais fatores que dificulta a capacidade das aves em se regular termicamente, durante o estresse por calor (MASHALY et al., 2004).

Conforme o modelo da figura 1-B, pode-se observar que a umidade relativa do ar pode afetar negativamente a produção de ovos das poedeiras da raça PRB quando for superior a $85,9 \%$. Segundo VERCESE (2010), a adaptação genética é um conjunto de alterações herdáveis das características que favorecem a sobrevivência de uma população de indivíduos em determinado ambiente, podendo envolver modificações evolutivas em muitas gerações (seleção natural) ou aquisição de propriedades genéticas específicas (seleção artificial). Os resultados observados (Figura 1-B e C) diferem entre os genótipos estudados e podem ser relacionados às diferenças na aclimatação (MAHMOUD \& YASEEN, 2005). Segundo a UPS THERMAL COMMISSION (2001), a aclimatação também pode ser especificada como uma adaptação fenotípica particular ou uma condição geneticamente fixa de uma espécie, subespécie ou sua evolução, que favorece a sobrevivência e ou melhore a produtividade em um determinado ambiente, podendo ocorrer em aves. 
Aves das raças RIR e PRB, quando submetidas a mais de um dia com temperatura acima de $32^{\circ} \mathrm{C}$, produzem ovos com peso superior ao esperado e, nessas mesmas condições, as aves PRW apresentam o peso dos ovos normal (Figura 1-C). Na figura 1-C, pode-se observar que as aves das três raças com idade inferior a 29 semanas de idade submetidas a mais de dois dias de frio com temperaturas inferiores a $10^{\circ} \mathrm{C}$ apresentaram peso de ovo inferior ao esperado. Esses resultados estão de acordo com os encontrados por SAHIN et al. (2002), que, comparando dois grupos de aves mantidas em diferentes temperaturas $\left(18^{\circ} \mathrm{C} \mathrm{e} 6^{\circ} \mathrm{C}\right)$, observaram que a produção e a qualidade dos ovos foram reduzidos em estresse por baixa temperatura. A temperatura ambiente baixa provoca alguns efeitos adversos no desempenho das aves, incluindo aumento da ingestão de alimentos, diminuição da produção de ovos e piora na digestibilidade de nutrientes (SPINU \&DEGEN, 1993).

\section{CONCLUSÃO}

A Mineração de Dados permite estabelecer características chave para classificar as raças de poedeiras, podendo estabelecer limites para aumentar a pressão de seleção em programas de melhoramento genético. A taxa de postura na 25 a semana e o peso de ovo na $33^{\mathrm{a}}$ semana de idade são os principais atributos relacionados com a classificação zootécnica das raças estudadas. Através da mineração de dados, pode-se identificar fatores ambientais que influenciam nas características fenotípicas de aves de diferentes genótipos. Poedeiras PRW apresentam na fase de prépico uma maior produção de ovos em relação às demais raças estudadas. As poedeiras PRW são mais sensíveis ao calor que as RIR e PRB, o que interfere negativamente na produção de ovos.

\section{REFERÊNCIAS}

BERRY, M.J.A.; LINOFF, G. Data mining techniques for marketing, sales and customer support. Indiana: Wiley Computer Publishing, 1997. 454p.

DEKKERS, J.C.M. Breeding in the 21th century: application of molecular technology. Proceedings of the Association for the Advancement of the Animal Breeding and Genetics, v.13, p.1-16, 1999.

FAYYAD, U.; STOLORZ, P. Data mining and KDD: promise and challenges. Future Generation Computer Systems, v.13, p.99-115, 1997.

HAGGER, C. Genetic correlations between body weight of cocks and production traits in laying hens, and their possible use in breeding schemes. Poultry Science, Champaign, v.73, n.3, p.381-387, 1994. Disponível em: <http://ps.fass.org/ content/73/3.toc $>$. Acesso em: 02 mar. 2011. doi: 10.3382/ ps.0730381.

LAVRAC, N. Machine learning for data mining in medicine. Lecture Notes in Computer Science, v.1620, p.47-62, 1999.

LIMA, M.G.F.; RODRIGUES, L.H.A. Árvore de decisão aplicada em dados de incubação de matrizes de postura hy-line W36. Ciência Agrotécnica, v.34, n.6, p.1550-1556, 2010. Disponível em: <http://www.editora.ufla.br/site/_adm/upload/ revista/34-6-2010_28.pdf>. Acesso em: 15 set. 2011.

MAHMOUD, K.Z.; YASEEN, A.M. Effect of feed withdrawal and heat acclimatization on stress responses of male broiler and layer-type chickens (Gallus gallus domesticus). AsianAustralian Journal of Animal Science, v.18, n.10, p.14451450, 2005. Disponível em: <http://www.ajas.info/Editor/ manuscript/upload/18_225.pdf>. Acesso em: 03 out. 2011.

MASHALY, M.M. et al. Effect of heat stress on production parameters and immune responses of commercial laying hens. Poultry Science, v.83, p.889-894, 2004. Disponível em: <http://ps.fass.org/content/83/6/889.full.pdf + html>. Acesso em: 04 out. 2011.

MITRA, S.S.; MITRA, P. Data mining in soft computing framework: a survey. IEEE Transactions on Neural Networks, v.13, n.1, p.3-14, 2002.

NÄÄS, I.A. et al. Impact of global warming on beef cattle production cost in Brazil. Scientia Agrícola, v.67, n.1, p.18, 2010. Disponível em: <http://www.scielo.br/scielo.php ?script=sci_arttext\&pid=S010390162010000100001\&lng=en $\&$ nrm=iso\&tlng=en>. Acesso em: 05 nov. 2011. doi: 10.1590/ S0103-90162010000100001.

NARDONE, A. et al. Climatic effects on productive traits in livestock. Veterinary Research Communications, v.30, n. 1, p.75-81, 2006.

PEREIRA, D.F. et al. Estimating mortality in laying hens as the environmental temperature increases. Brazilian Journal of Poultry Science, v.12, n.4, p. 265-271, 2010. Disponível em: <http://www.scielo.br/scielo.php?pid=S1516635X2010 000400008\&script=sci_abstract $>$. Acesso em: 06 jul. 2011. doi: org/10.1590/S1516-635X2010000400008.

PIASSI, M. et al. Estudo da divergência genética entre oito grupos genéticos de aves de postura, por meio de técnicas de análise multivariada. Revista Brasileira de Zootecnia, v.24, p.715-727, 1995.

POGGENPOEL, D.G. et. al. Genetic basis of the increase in egg weight with pullet age in a White Leghorn flock. British Poultry Science, v.37, n.4, p.743-756, 1996. Disponível em: <http://www.tandfonline.com/doi/abs/10.1080/000716688 08417115\#preview>. Acesso em: fev. 2012. doi: 10.1080/ 00071668808417115.

QUINLAN, J.R. C4.5: Programs for machine learning. San Mateo: Morgan Kaufmann Publishers, 1993. 302p.

QUINLAN, J.R. Improved use of continuous attributes in C4.5. Journal of Artificial Intelligence Research, v.4, p.77-90, 1996. 
SAHIN, K. et al. Effects of dietary chromium picolinate and ascorbic acid supplementation on egg production, egg quality and some blood metabolites of laying hens reared under low ambient temperature (6 degrees C). Archives Tieremahr, v.56, p.41-49, 2002.

SPINU, M.; DEGEN, A.A. Effect of cold stress on performance and immune responses of bedouin and white leghorn hens. British Poultry Science, v.34, p.177-181, 1993.

UPS THERMAL COMMISSION. Glossary of terms for thermal physiology. Japanese Journal of Physiology, v.51, p.245-280, 2001.

VALE, M.M. et al. Data mining to estimate broiler mortality when exposed to heat wave. Scientia Agricola, v.65, n.3, p.223-229, 2008.
VALE, M.M. et al. Heat waves characterization with impact over broilers mortality rates between 29 days old at the slaughter. Brazilian Journal of Poultry Science, v.12, n.4, p.279$285,2010$.

VERCESE, F. Efeito da temperatura sobre o desempenho e a qualidade dos ovos de Codornas Japonesas. 2010. 59p. Dissertação (Mestrado em Zootecnia) - Universidade Estadual Paulista, Faculdade de Medicina Veterinária e Zootecnia, Botucatu, SP.

WITTEN, I.H.; FRANK, E. Data mining: practical machine learning tools and techniques. 2.ed. San Francisco: Morgan Kaufmann, 2005. 525p. 